\title{
Fringe integral equation method for a truncated grounded dielectric slab
}

\author{
Jørgensen, Erik; Maci, S.; Toccafondi, A.
}

Published in:

I E E E Transactions on Antennas and Propagation

Link to article, DOI:

$10.1109 / 8.943316$

Publication date:

2001

Document Version

Publisher's PDF, also known as Version of record

Link back to DTU Orbit

Citation (APA):

Jørgensen, E., Maci, S., \& Toccafondi, A. (2001). Fringe integral equation method for a truncated grounded dielectric slab. I E E E Transactions on Antennas and Propagation, 49(8), 1210-1217.

https://doi.org/10.1109/8.943316

\section{General rights}

Copyright and moral rights for the publications made accessible in the public portal are retained by the authors and/or other copyright owners and it is a condition of accessing publications that users recognise and abide by the legal requirements associated with these rights.

- Users may download and print one copy of any publication from the public portal for the purpose of private study or research.

- You may not further distribute the material or use it for any profit-making activity or commercial gain

- You may freely distribute the URL identifying the publication in the public portal

If you believe that this document breaches copyright please contact us providing details, and we will remove access to the work immediately and investigate your claim. 


\title{
Fringe Integral Equation Method for a Truncated Grounded Dielectric Slab
}

\author{
Erik Jørgensen, Student Member, IEEE, Stefano Maci, Senior Member, IEEE, and Alberto Toccafondi, Member, IEEE
}

\begin{abstract}
The problem of scattering by a semi-infinite grounded dielectric slab illuminated by an arbitrary incident $\mathbf{T} \mathbf{M}_{z}$ polarized electric field is studied by solving a new set of "fringe" integral equations (F-IEs), whose functional unknowns are physically associated to the wave diffraction processes occuring at the truncation. The F-IEs are obtained by subtracting from the surface/surface integral equations pertinent to the truncated slab, an auxiliary set of equations obtained for the canonical problem of an infinite grounded slab illuminated by the same source. The F-IEs are solved by the method of moments by using a set of subdomain basis functions close to the truncation and semi-infinite domain basis functions far from it. These latter functions are properly shaped to reproduce the asymptotic behavior of the diffracted waves, which is obtained by physical inspection. The present solution is applied to the case of an electric line source located at the air-dielectric interface of the slab. Numerical results are compared with those calculated by a physical optics approach and by an alternative solution, in which the integral equation is constructed from the field continuity through an aperture orthogonal to the slab. The applications of the solution to an array of line currents are also presented and discussed.
\end{abstract}

Index Terms-Fringe integral equation.

\section{INTRODUCTION}

$\mathbf{T}$ HE description of diffraction mechanisms occurring at edges in a truncated grounded dielectric slab is of importance in various engineering applications, principally regarding patch antennas. Spurious diffraction waves may considerably affect side lobes and cross-polar levels of the radiation pattern [1], [2], especially when surface waves are strongly excited. However, diffraction mechanisms excited by the truncation are not very well investigated in the literature by exact approaches. Indeed, the canonical reference solution of a grounded semi-infinite dielectric slab-or, more generally, that of a dielectric wedge-is not yet available in analytical form, and the application of a full-wave numerical analysis appears quite cumbersome owing to the infinite extension of the canonical structure. The use of approximate boundary conditions (BCs) [3] like impedance $\mathrm{BC}$ or generalized impedance $\mathrm{BC}$ [4] is often used to obtain a canonical solution from which high-frequency diffraction coefficients are derived through asymptotic evaluation. These solutions, however, fail for increasing substrate thicknesses and for low dielectric constants, due to the inaccuracy of the impedance $\mathrm{BC}$ approximations,

Manuscript received March 17, 1999; revised September 14, 2000.

E. Jørgensen is with the Department of Electromagnetic Systems, Technical University of Denmark, Lyngby DK-2800 Denmark.

S. Maci and A. Toccafondi are with the Department of Information Engineering, University of Siena, Siena 53100 Italy.

Publisher Item Identifier S 0018-926X(01)01837-3. especially when the exciting source is located at the boundary. In most practical instances, they cannot be applied to patch antenna problems. For values of thickness and dielectric constants typically involved in these problems, a physical optics (PO) approach seems to be quite adequate [5]. This approximation consists of estimating the radiating currents on the semi-infinite substrate from those produced by the infinite structure. This has the impairment of neglecting guided wave reflections at the slab truncation. However, this phenomenon is presumably less important for low permittivity substrates, such as those used in patch antennas.

The construction of a reference solution by solving integral equations may be extremely useful to validate the effectiveness and the accuracy of the aforementioned PO or generalized impedance BC approximations. To this end, an appropriate canonical structure is considered that consists of a semi-infinite grounded slab illuminated by a two-dimensional source. This allows a clear interpretation of the single diffraction mechanisms excited by space waves and guided waves, with the consequent possibility to validate diffraction coefficients developed by high-frequency approximations. Both differential equation and integral equation methods may be used to perform the full-wave analysis of this kind of structures. Both of these classes of methods suffer from the infiniteness of the structure. A time-domain differential method like finite difference time domain (FDTD) may overcome this limitation by isolating the diffraction effects by time gating, thus reducing a finite structure to a virtually semi-infinite one. However, the conspicuous computation time and the difficulty in controlling the accuracy often render it preferable to resort to integral equation methods in the frequency domain. In [6], a surface/surface integral equation for a dielectric loaded wedge illuminated by a plane wave was solved with the method of moments (MoM) by using an appropriate expansion of the unknown currents in terms of entire domain basis functions. These latter were shaped in order to account for the asymptotic behavior of the edge diffracted field. This approach was used to check the validity of the impedance BC. However, its extension to a more general near-source illumination is not a straightforward matter.

Plane wave illumination is also assumed in [7], but the integral equation there represents the continuity of the electric field at an infinite aperture perpendicular to the slab and passing trough the edge. In that work, the unknown currents are represented only in terms of subdomain basis functions, so the final results are affected by truncation errors. The same aperture integral equation (A-IE) concept has been extended to a more general source excitation by different authors independently [8], [9], with the inclusion of entire domain basis functions that 
properly account for the infinite extension of the space domain on which the IE is applied. We note that although the work in [8] and [9] is seen on different perspectives, the two formulations are substantially equivalent.

In this paper, we present a full-wave analysis of a truncated grounded dielectric slab illuminated by an arbitrary incident transverse magnetic field. This method is based on the formulation of a set of "fringe" integral equations (F-IEs) obtained by subtracting from the surface/surface integral equation pertinent to the semi-infinite slab, an auxiliary set of equations obtained for the canonical problem of an infinite slab illuminated by the same incident field. This allows us to interpret the unknown functions of the F-IEs in terms of contributions that can be physically associated to the diffraction phenomena occurring at the truncation. Taking advantage of this interpretation, a MoM solution is obtained with pulse basis functions localized on a finite region near the truncation and semi-infinite domain basis functions to reproduce the edge-excited surface waves far from the edge. To speed up the convergence, an additional semi-infinite domain basis function is included for describing the space wave diffracted field. This formulation is then applied to calculate the field scattered by an electric line source placed at the air-dielectric interface.

Before proceeding further, it is worth spending some words on the difference between the A-IE method [7]-[9] and the F-IE method presented here. As mentioned above, A-IE is obtained by applying the continuity of the electric field through an infinite aperture perpendicular to the slab passing at the edge. This formulation employs both the Green's function of the slab and of the free space. The A-IE method may be applied in conjunction with the extraction of the geometrical optics field at the aperture. Although this extraction appears quite cumbersome for near-source excitation, this would make the A-IE method conceptually similar to the F-IE method presented here. However, due to the use of the slab Green's function, the A-IE approach is more time-consuming. Furthermore, the method presented here provides separation of space wave diffraction and surface wave diffraction contributions, thus allowing the isolation of numerical diffraction coefficients associated to each individual physical mechanism. The same separation is not possible in the A-IE method.

This paper is organized as follows. In Section II, the surface/surface integral equation for a truncated slab is presented. Section III is devoted to developing the fringe integral equations. In Section IV, a MoM solution for these equations is formulated. The application of the proposed method to the problem of an electric line source placed at the air-dielectric interface is discussed in Section V. In Section VI, numerical calculations and comparisons with both the A-IE method [8] and a generalized PO approach [5] are presented and discussed. Conclusions and future developments are drawn in Section VII.

\section{Surface/Surface InTEGRAL EQUATIONS}

Consider the semi-infinite grounded dielectric slab in Fig. 1. The dielectric substrate has thickness $h$ and dielectric permittivity $\epsilon_{1}=\epsilon_{r} \epsilon_{0}$. A rectangular coordinate system is introduced with the $x$-axis oriented orthogonal to the edge and along the

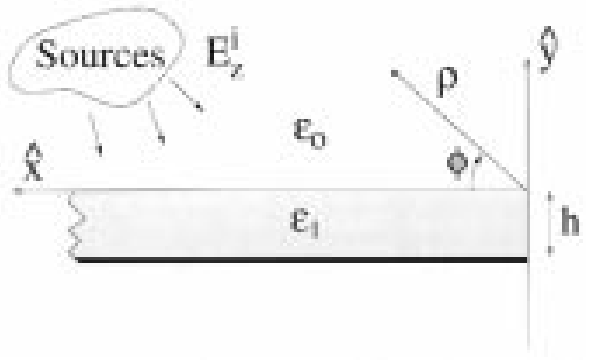

Fig. 1. Semi-infinite grounded dielectric slab.

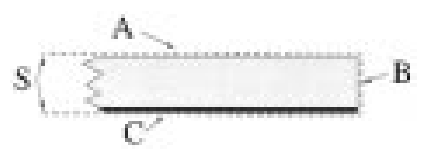

Fig. 2. Surface for application of equivalence principle.

top of the substrate, the $y$-axis orthogonal to the slab, and the $\hat{z}$-axis along the truncation. Let us assume the truncated slab is excited by a system of $z$-invariant sources that creates only a $\hat{z}$-directed incident electric field ( $\mathrm{TM}_{z}$ polarization). The following formulation is carried out with these restrictions, but a linear phase variation along $z$ can be introduced without conceptual efforts. For a $\mathrm{TE}_{z}$ polarized source, the procedure presented here is still valid, although a higher number of unknowns is needed for describing the edge singularity. In order to derive the proper integral equations, we choose a surface $S$ to apply the equivalence principle. As shown in Fig. 2, the surface $S$ envelopes the boundary of the slab passing on the back of the ground plane. We denote with $A$ and $B$ the portions of $S$ that are on top of the substrate and on the external truncation side, respectively, and with $C$ that on the back of the ground plane. The first step of our procedure is an application of the surface/surface IE method [10].

\section{A. The Exterior Problem}

In order to determine the field $\left(\mathbf{E}^{\text {ext }}, \mathbf{H}^{\text {ext }}\right)$ in the region outside $S$, the region inside $S$ is filled with zero field and free space. The continuity of the tangential electric field is reconstructed by distributing equivalent electric and magnetic surface currents on $A\left(\mathbf{J}_{A}, \mathbf{M}_{A}\right)$ and $B\left(\mathbf{J}_{B}, \mathbf{M}_{B}\right)$ and an electric surface current on $C\left(\mathbf{J}_{C}\right)$, as shown in Fig. 3. We now introduce the notation $\mathbf{E}_{0}[\mathbf{J}, \mathbf{M}]$ to denote the electric field radiated in free space by the pair $(\mathbf{J}, \mathbf{M})$. Explicit expressions for the integral operator $\mathbf{E}_{0}[\mathbf{J}, \mathbf{M}]$ can be found in the Appendix. The equivalence principle applied on $A$ and $B$ yields

$$
\begin{aligned}
& \mathbf{E}^{i}(\boldsymbol{\rho})+\mathbf{E}_{0}\left[\mathbf{J}_{A}^{\mathrm{ext}}, \mathbf{M}_{A}^{\mathrm{ext}}\right]+\mathbf{E}_{0}\left[\mathbf{J}_{B}^{\mathrm{ext}}, \mathbf{M}_{B}^{\mathrm{ext}}\right]+\mathbf{E}_{0}\left[\mathbf{J}_{C}^{\mathrm{ext}}, 0\right] \\
& =\hat{n} \times \mathbf{M}_{A, B}^{\mathrm{ext}}(\boldsymbol{\rho}), \quad \boldsymbol{\rho} \in A, B
\end{aligned}
$$

where $\rho$ denotes an observation point on $A$ or $B$, and $\hat{n}$ is the outward unit vector normal to $S$. Vanishing of the tangential electric field on $C$ yields

$$
\begin{aligned}
& \mathbf{E}^{i}(\boldsymbol{\rho})+\mathbf{E}_{0}\left[\mathbf{J}_{A}^{\mathrm{ext}}, \mathbf{M}_{A}^{\mathrm{ext}}\right]+\mathbf{E}_{0}\left[\mathbf{J}_{B}^{\mathrm{ext}}, \mathbf{M}_{B}^{\mathrm{ext}}\right]+\mathbf{E}_{0}\left[\mathbf{J}_{C}^{\mathrm{ext}}, 0\right] \\
& \quad=0, \quad \boldsymbol{\rho} \in C .
\end{aligned}
$$




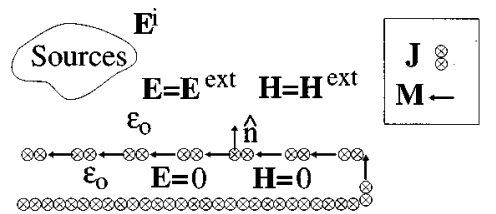

Fig. 3. Exterior problem.

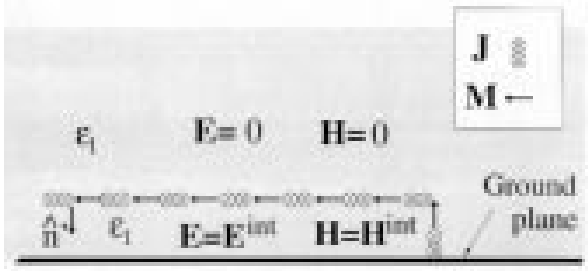

Fig. 4. Interior problem.

\section{B. The Interior Problem}

In order to find the total field $\left(\mathbf{E}^{\text {int }}, \mathbf{H}^{\text {int }}\right)$ inside $S$, we postulate zero field in the exterior region, which is supposed to be filled with the dielectric material of the slab and with the virtual continuation of the ground plane. Electric and magnetic surface currents are distributed on $A$ and $B$, which radiate in a homogeneous dielectric region bounded by an infinite ground plane (Fig. 4). Next, use of the image principle leads to the original currents plus their images through the infinite ground plane radiating in a fully homogeneous dielectric. The tangential electric field radiated in this medium by both $(\mathbf{J}, \mathbf{M})$ and their images is denoted with $\mathbf{E}_{1}^{*}[\mathbf{J}, \mathbf{M}]$. The equivalence principle applied on $A$ and $B$ leads to

$$
\begin{gathered}
\mathbf{E}_{1}^{*}\left[\mathbf{J}_{A}^{\mathrm{int}}, \mathbf{M}_{A}^{\mathrm{int}}\right]+\mathbf{E}_{1}^{*}\left[\mathbf{J}_{B}^{\mathrm{int}}, \mathbf{M}_{B}^{\mathrm{int}}\right] \\
\quad=\hat{n}^{\prime} \times \mathbf{M}_{A, B}^{\mathrm{int}}(\boldsymbol{\rho}), \quad \boldsymbol{\rho} \in A, B
\end{gathered}
$$

where $\hat{n}^{\prime}$ is the inward unit vector normal to $S$.

\section{Coupled Integral Equations}

The exterior and the interior problem are now combined by imposing the continuity of the tangential electric and magnetic fields on $A$ and $B$

$$
\begin{aligned}
\hat{n} \times \mathbf{H}_{A, B}^{\mathrm{int}} & =\hat{n} \times \mathbf{H}_{A, B}^{\mathrm{ext}} \Rightarrow \mathbf{J}_{A, B}^{\mathrm{int}}=-\mathbf{J}_{A, B}^{\mathrm{ext}} \\
\mathbf{E}_{A, B}^{\mathrm{int}} \times \hat{n} & =\mathbf{E}_{A, B}^{\mathrm{ext}} \times \hat{n} \Rightarrow \mathbf{M}_{A, B}^{\text {int }}=-\mathbf{M}_{A, B}^{\text {ext }} .
\end{aligned}
$$

Introducing (4) and (5) in (1)-(3) leaves only the unknown current components $\mathbf{J}_{A}, \mathbf{J}_{B}, \mathbf{J}_{C}, \mathbf{M}_{A}$, and $\mathbf{M}_{B}$ defined for the exterior problem, where we have suppressed and understood the superscript ext. The resulting coupled equations are not yet appropriate for solving directly by MoM, due to the infinite dimension of the surface $S$. As a consequence, an auxiliary set of equations are derived next.

\section{F-IEs}

We now consider the infinite grounded dielectric slab (Fig. 5) illuminated by the same sources as the truncated configuration. The complementary surface to $A$ on the top of the substrate is denoted with $D$ [Fig. 5(a)]. For this problem, the exact solution

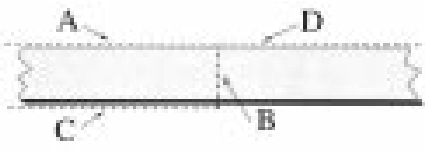

(a)

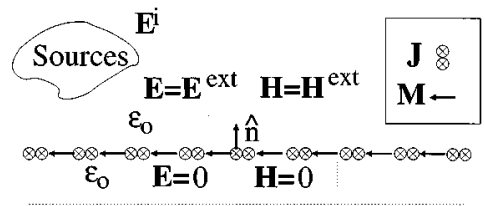

(b)

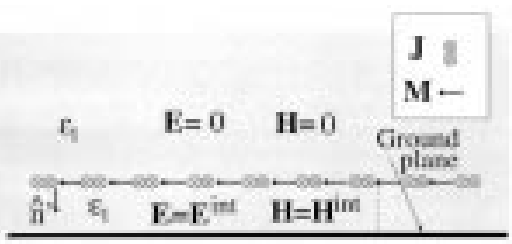

(c)

Fig. 5. Infinite grounded slab (a) surface for application of the equivalence principle, (b) exterior problem, and (c) interior problem.

is known in analytical form. However, the information we may extract from this solution allow us to build up a new set of integral equations. To this end, let us apply the equivalence principle on $A+D$, using the same procedure as that for the truncated structure. This yields for the exterior problem [Fig. 5(b)]

$$
\begin{aligned}
& \mathbf{E}^{i}(\boldsymbol{\rho})+\mathbf{E}_{0}\left[\mathbf{J}_{A}^{\mathrm{PO}}, \mathbf{M}_{A}^{\mathrm{PO}}\right]+\mathbf{E}_{0}\left[\mathbf{J}_{D}^{\mathrm{PO}}, \mathbf{M}_{D}^{\mathrm{PO}}\right] \\
& \quad=\hat{n} \times \mathbf{M}_{A}^{\mathrm{PO}}(\boldsymbol{\rho}), \quad \boldsymbol{\rho} \in A \\
& \mathbf{E}^{i}(\boldsymbol{\rho})+\mathbf{E}_{0}\left[\mathbf{J}_{A}^{\mathrm{PO}}, \mathbf{M}_{A}^{\mathrm{PO}}\right]+\mathbf{E}_{0}\left[\mathbf{J}_{D}^{\mathrm{PO}}, \mathbf{M}_{D}^{\mathrm{PO}}\right] \\
& \quad=0, \quad \boldsymbol{\rho} \in B, C .
\end{aligned}
$$

For the interior problem [Fig. 5(c)], we get

$$
\begin{gathered}
-\mathbf{E}_{1}^{*}\left[\mathbf{J}_{A}^{\mathrm{PO}}, \mathbf{M}_{A}^{\mathrm{PO}}\right]-\mathbf{E}_{1}^{*}\left[\mathbf{J}_{D}^{\mathrm{PO}}, \mathbf{M}_{D}^{\mathrm{PO}}\right] \\
\quad=\hat{n} \times \mathbf{M}_{A, B}^{\mathrm{PO}}(\boldsymbol{\rho}), \quad \boldsymbol{\rho} \in A, B .
\end{gathered}
$$

We have used the superscript PO, since PO currents and fields are exact solutions of the infinite problem. We now subtract the equations relevant to the infinite structure (6)-(8) from the corresponding equations for the finite structure (1)-(3). Introducing the convenient quantities $\tilde{\mathbf{X}}=\mathbf{X}-\mathbf{X}^{\mathrm{PO}}$, where $\mathbf{X}=\mathbf{J}, \mathbf{M}$, and using the linearity of the integral operators $\mathbf{E}_{0}[\mathbf{J}, \mathbf{M}]$ and $\mathbf{E}_{1}^{*}[\mathbf{J}, \mathbf{M}]$ leads to

$$
\begin{aligned}
& -\hat{n} \times \tilde{\mathbf{M}}_{A}(\boldsymbol{\rho})+\mathbf{E}_{0}\left[\tilde{\mathbf{J}}_{A}, \tilde{\mathbf{M}}_{A}\right]+\mathbf{E}_{0}\left[\mathbf{J}_{B}, \mathbf{M}_{B}\right]+\mathbf{E}_{0}\left[\mathbf{J}_{C}, 0\right] \\
& \quad=\mathbf{E}_{0}\left[\mathbf{J}_{D}^{\mathrm{PO}}, \mathbf{M}_{D}^{\mathrm{PO}}\right], \quad \boldsymbol{\rho} \in A \\
& -\hat{n} \times \mathbf{M}_{B}(\boldsymbol{\rho})+\mathbf{E}_{0}\left[\tilde{\mathbf{J}}_{A}, \tilde{\mathbf{M}_{A}}\right]+\mathbf{E}_{0}\left[\mathbf{J}_{B}, \mathbf{M}_{B}\right]+\mathbf{E}_{0}\left[\mathbf{J}_{C}, 0\right] \\
& \quad=\mathbf{E}_{0}\left[\mathbf{J}_{D}^{\mathrm{PO}}, \mathbf{M}_{D}^{\mathrm{PO}}\right], \quad \boldsymbol{\rho} \in B \\
& \mathbf{E}_{0}\left[\tilde{\mathbf{J}}_{A}, \tilde{\mathbf{M}}_{A}\right]+\mathbf{E}_{0}\left[\mathbf{J}_{B}, \mathbf{M}_{B}\right]+\mathbf{E}_{0}\left[\mathbf{J}_{C}, 0\right] \\
& \quad=\mathbf{E}_{0}\left[\mathbf{J}_{D}^{\mathrm{PO}}, \mathbf{M}_{D}^{\mathrm{PO}}\right], \quad \boldsymbol{\rho} \in C \\
& \hat{n} \times \tilde{\mathbf{M}}_{A}(\boldsymbol{\rho})+\mathbf{E}_{1}^{*}\left[\tilde{\mathbf{J}}_{A}, \tilde{\mathbf{M}}_{A}\right]+\mathbf{E}_{1}^{*}\left[\mathbf{J}_{B}, \mathbf{M}_{B}\right] \\
& \quad=\mathbf{E}_{1}^{*}\left[\mathbf{J}_{D}^{\mathrm{PO}}, \mathbf{M}_{D}^{\mathrm{PO}}\right], \quad \boldsymbol{\rho} \in A \\
& \hat{n} \times \mathbf{M}_{B}(\boldsymbol{\rho})+\mathbf{E}_{1}^{*}\left[\tilde{\mathbf{J}}_{A}, \tilde{\mathbf{M}}_{A}\right]+\mathbf{E}_{1}^{*}\left[\mathbf{J}_{B}, \mathbf{M}_{B}\right] \\
& \quad=\mathbf{E}_{B}^{\mathrm{PO}}(\boldsymbol{\rho})+\mathbf{E}_{1}^{*}\left[\mathbf{J}_{D}^{\mathrm{PO}}, \mathbf{M}_{D}^{\mathrm{PO}}\right], \quad \boldsymbol{\rho} \in B .
\end{aligned}
$$


In (9)-(13), the terms containing unknown quantities are written on the left side and the known terms on the right side. At a first glance, these five coupled equations look more complicated than the original ones. However, they are very convenient when solving by MoM, since the unknowns are exclusively associated to mechanisms generated by the truncation. In particular, both $\tilde{\mathbf{J}}_{A}$ and $\tilde{\mathbf{M}}_{A}$ (i.e., the difference between the exact currents of the finite slab and those of the infinite one) contain two contributions: 1) space waves diffracted at the top edge and 2) guided waves (of surface or leaky type) excited by the truncation, when these latter are supported by the slab. The unknown $\mathbf{J}_{C}$ is the induced electric surface current on the back side of the ground plane, which only consists of a space wave contribution diffracted at the edge of the ground plane. Note that the unknowns $\mathbf{J}_{B}$ and $\mathbf{M}_{B}$ are the same quantities as of the original set of IEs, but since they are localized on a small portion of $S$, no problems occur in their description in a MoM scheme. The right sides of (9)-(13) are the forcing terms of the F-IEs and can be found by integration of the electric and magnetic PO surface currents on $D$. As apparent by (6)-(8), these forcing terms can also be determined by using $A$ as integration domain. However, this introduces numerical problems when sources are located exactly at the surface $A$. Indeed, in this case, the PO currents lead to a field singularity at the source, which must cancel that of the incident field, thus comprising certain complications when calculating the forcing terms. Furthermore, using $D$ as integration domain provides forcing fields that exhibit an evident diffractive nature, being asymptotically dominated by the end-point radiation. This leaves open the possibility to apply asymptotic evaluations.

The procedure presented above allows one to solve exclusively for the current components introduced by the truncation. Except for the surface wave contribution to $\tilde{\mathbf{J}}_{A}$ and $\tilde{\mathbf{M}}_{A}$, which will be treated separately, all the unknowns used in (9)-(13) exhibit a behavior localized in a narrow region in vicinity of the edge. Since we solve for the fringe currents alone, we will denote (9)-(13) by F-IEs, in analogy with the terminology of the physical theory of diffraction [11]

\section{MoM SoluTiON}

In this section, we solve the coupled (9)-(13) by using a MoM scheme. In a region close to the truncation, we expand the unknown currents in terms of subdomain pulse functions $P^{m}$ given by

$$
P^{n}(\boldsymbol{\rho})= \begin{cases}1, & \boldsymbol{\rho} \in S^{n} \\ 0, & \text { otherwise }\end{cases}
$$

where $S^{n}$ is the $n$th segment. We will refer to this finite region as the subdomain region (SR). On the part of the surface $A$ that is located in the SR, we use the expansion

$$
\tilde{X}_{A}(\boldsymbol{\rho})=\sum_{n=1}^{N_{A}} \tilde{X}_{A}^{n} P^{n}(\boldsymbol{\rho}), \quad \boldsymbol{\rho} \in A, \quad X=\mathbf{J}, \mathbf{M}
$$

where

$$
N_{A} \quad \text { total number of segments on } A \text {; }
$$$$
\tilde{X}_{A}(\boldsymbol{\rho}) \quad \tilde{\mathbf{J}}_{A}(\boldsymbol{\rho}) \text { or } \tilde{\mathbf{M}}_{A}(\boldsymbol{\rho}) \text {; }
$$

$\tilde{X}_{A}^{n} \quad$ unknown expansion coefficients to be determined by MoM.

Similarly, on $B$ and on the portion of $C$ that lies inside the SR, we get the expansions

$$
X_{B, C}(\boldsymbol{\rho})=\sum_{n=1}^{N_{B, C}} X_{B, C}^{n} P^{n}(\boldsymbol{\rho})
$$

where $X=\mathbf{J}, \mathbf{M}$ when $\boldsymbol{\rho} \in B$ and $X=\mathbf{J}$ when $\boldsymbol{\rho} \in C$.

Outside the SR, we use the asymptotic behavior of the fields to define a set of expansion functions with semi-infinite domains. As mentioned earlier, the unknowns $\tilde{\mathbf{J}}_{A}$ and $\tilde{\mathbf{M}}_{A}$ comprise contributions of a space wave diffracted at the edge, and possibly one or more guided waves excited at the truncation. The space wave diffracted field asymptotically propagates with the speed of light and decays like $1 /(\rho \sqrt{\rho})$. At variance, the surface waves (SWs) propagate unattenuated with a phase velocity between the speed of light in free space and that in the dielectric. To describe the asymptotic field behavior outside the SR, we then use the expansion

$$
\tilde{X}_{A}(\rho)=\tilde{X}_{A}^{N_{A}+1} \frac{e^{-j k_{0} \rho}}{\rho \sqrt{\rho}}+\tilde{X}_{A}^{N_{A}+2} e^{-j k_{\mathrm{sw}} \rho}, \quad \rho \in A
$$

where $X=\mathbf{J}, \mathbf{M}, k_{\mathrm{sw}}$ is the $\mathrm{SW}$ wavenumber and $\tilde{X}_{A}^{N_{A}+1}$ and $\tilde{X}_{A}^{N_{A}+2}$ can be interpreted as the unknown diffraction coefficient of the space wave and the excitation coefficient of the SW, respectively. In (17), we have assumed that only the first SW mode is propagating. This contribution must be left out if SW propagation is not supported by the structure, or similar terms (with the proper $k_{\mathrm{Sw}}$ ) must be added when more than one $\mathrm{SW}$ is supported. Note that possible leaky modes excited at the truncation are exponentially attenuated toward positive $x$, and their description can be entirely attributed to the pulse functions in the SR. The unknown quantity on the surface $C$ is the induced surface current $J_{C}$ on the back side of the ground plane. Outside the SR, we use the expansion

$$
\mathbf{J}_{C}(\boldsymbol{\rho})=\mathbf{J}_{C}^{N_{C}+1} \frac{e^{-j k_{0} \rho}}{\rho \sqrt{\rho}}, \quad \boldsymbol{\rho} \in C
$$

where $\mathbf{J}_{C}^{N_{C}+1}$ can be interpreted as the unknown diffraction coefficient. Note that the spreading factor is of order $1 /(\rho \sqrt{\rho})$ due to the $\mathrm{TM}_{z}$ excitation. The weighting functions are chosen to be Dirac delta functions. When SWs are propagating, it is necessary to use one test point for each expansion function in the semi-infinite region, taking care to avoid superposition of test points. By using the segmentation described above, the total number of test points is $2\left(N_{A}+2\right)+2 N_{B}+\left(N_{C}+1\right)$.

\section{Application to the Electric Line Source Problem}

To demonstrate the application of the F-IE method presented above, we now consider the problem of an electric line source placed at the air-dielectric interface at $(x, y)=(L, 0)$. This case, as well as the complementary $\mathrm{TE}_{z}$ case of a magnetic line source (which can be treated with the same formulation), is strictly related to patch antenna problems. By using the spectral Green's function of the infinite slab excited by a unit electric 
line source, the spectral integral representation of the PO currents on the surface $D$ may be written as

$$
\begin{aligned}
\mathbf{J}^{\mathrm{PO}}\left(x^{\prime}\right) & =-\hat{z} \frac{1}{2 \pi} \int_{-\infty}^{\infty} e^{j k_{x}\left(x^{\prime}-L\right)} J\left(k_{x}\right) d k_{x} \\
\mathbf{M}^{\mathrm{PO}}\left(x^{\prime}\right) & =\hat{x} \frac{j \zeta_{0}}{2 \pi} \int_{-\infty}^{\infty} e^{j k_{x}\left(x^{\prime}-L\right)} M\left(k_{x}\right) d k_{x}
\end{aligned}
$$

where $k_{x}$ is the spectral wavenumber, $k_{y 0}=\sqrt{k_{0}^{2}-k_{x}^{2}}, k_{y 1}=$ $\sqrt{k_{1}^{2}-k_{x}^{2}}, \quad k_{1}=\sqrt{\epsilon_{r}} k_{0}$, and $\zeta_{0}$ is the free space impedance. Furthermore, $J\left(k_{x}\right)=k_{y 1} \cos \left(k_{y 1} h\right) / D\left(k_{x}\right)$ and $M\left(k_{x}\right)=k_{0} \sin \left(k_{y 1} h\right) / D\left(k_{x}\right)$, where $D\left(k_{x}\right)=$ $j k_{y 0} \sin \left(k_{y 1} h\right)+k_{y 1} \cos \left(k_{y 1} h\right)$. The change of variable $k_{x}=k_{0}\left(1-j s^{2}\right)$ with $k_{y 0}=-k_{0} s \sqrt{s^{2}+2 j}$ is now introduced. The original contour is deformed into the steepest descent path through the saddle point at $s=0$. This leads to

$$
\begin{aligned}
\mathbf{J}^{\mathrm{PO}}\left(x^{\prime}\right)= & -\hat{z} k_{0} G_{J}\left(x^{\prime}\right)-\hat{z} j k_{0} e^{j k_{0}\left(x^{\prime}-L\right)} \\
& \cdot \sum_{p} U\left[\operatorname{Im}\left(s_{p}\right)\right] e^{s_{p}^{2} k_{0}\left(x^{\prime}-L\right)} R_{J p} \\
\mathbf{M}^{\mathrm{PO}}\left(x^{\prime}\right)= & \hat{x} j k_{0} \zeta_{0} G_{M}\left(x^{\prime}\right)-\hat{x} k_{0} \zeta_{0} e^{j k_{0}\left(x^{\prime}-L\right)} \\
& \cdot \sum_{p} U\left[\operatorname{Im}\left(s_{p}\right)\right] e^{s_{p}^{2} k_{0}\left(x^{\prime}-L\right)} R_{M p}
\end{aligned}
$$

where $s_{p}$ is the $p$ th guided wave pole in the complex $s$-plane and $k_{0} R_{J p}$ and $k_{0} R_{M p}$ are the residues at the poles captured in the contour deformation. In (21) and (22), $U[\cdot]$ is the Heaviside unit step function and

$$
\begin{aligned}
& G_{X}\left(x^{\prime}\right)=\frac{e^{-j \Omega\left(x^{\prime}\right)}}{2 \pi}\left\{\int_{-\infty}^{\infty} e^{-s^{2} \Omega\left(x^{\prime}\right)} T_{X}(s) d s\right. \\
&\left.-\sqrt{\frac{\pi}{\Omega\left(x^{\prime}\right)}} \sum_{p} R_{X p} \frac{F\left[j \Omega\left(x^{\prime}\right) s_{p}^{2}\right]}{s_{p}}\right\} \\
& X=J, M
\end{aligned}
$$

in which $\Omega\left(x^{\prime}\right)=k_{0}\left|x^{\prime}-L\right|, F[\cdot]$ is the transition function of the uniform theory of diffraction (see [5] for its definition), and $T_{X}(s)$ is a regular function close to the saddle point $s=0$. This regular function is obtained by the Van der Waerden method [12] by extracting the pole singularities close to the saddle point as

$$
T_{X}(s)=B_{X}(s)-\sum_{p} \frac{R_{X p}}{s-s_{p}}, \quad X=J, M .
$$

In (24), $B_{J}(s)=-2 j s J\left(1-s^{2}\right)$ and $B_{M}(s)=-2 j s M(1-$ $\left.s^{2}\right)$. The number of poles to be extracted is determined by following the criterion suggested in [2]. The integral in (23) is easily evaluated numerically, since the smooth integrand exhibits a very rapid exponential decay around $s=0$. When $\Omega\left(x^{\prime}\right)$ is large, we replace $T_{X}(s)$ with the first two terms of its Taylor expansion around the saddle point at $s=0$. This leads to the

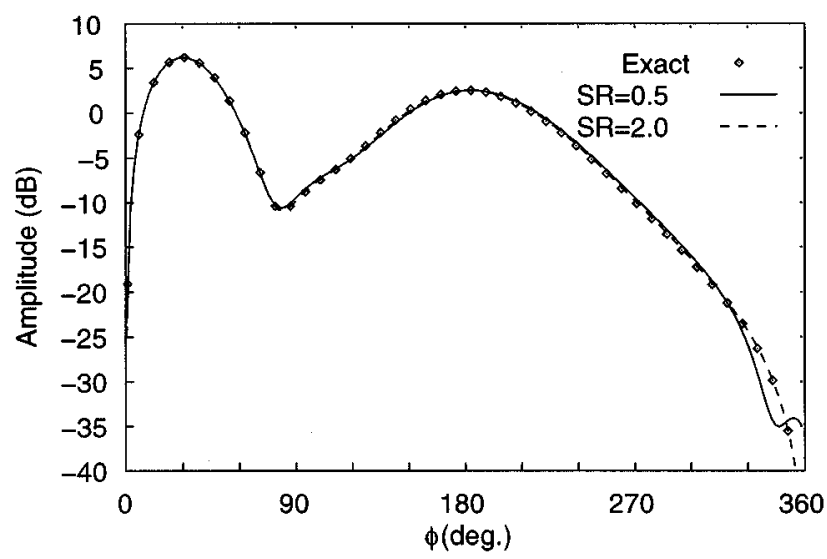

Fig. 6. $L=0.1 \lambda_{0}, h=0.5 \lambda_{0}, \epsilon_{r}=1.0$ (half-plane problem).

uniform asymptotic expression

$$
\begin{array}{r}
G_{X}\left(x^{\prime}\right) \sim \frac{e^{-j \Omega\left(x^{\prime}\right)}}{2 \sqrt{\pi \Omega\left(x^{\prime}\right)}}\left\{\left[T_{X}(0)+\frac{T_{X}^{\prime \prime}(0)}{4 \Omega\left(x^{\prime}\right)}\right]\right. \\
\left.-\sum_{p} R_{X p} \frac{F\left[j \Omega\left(x^{\prime}\right) s_{p}^{2}\right]}{s_{p}}\right\} \\
X=J, M .
\end{array}
$$

To obtain the forcing terms in (9)-(13), (21) and (22) must be multiplied with the pertinent Green's function of the homogeneous medium and integrated for $x^{\prime}$ ranging from $-\infty$ to zero. As suggested in [6], this latter integration on a semi-infinite domain is efficiently carried out with the method of averages [13]. For all values of $\Omega\left(x^{\prime}\right)$, the PO surface currents are calculated in a finite number of points, and quadratic interpolation is used for all remaining points of the integrand. The method of averages is also used in the calculation of the MoM impedance matrix, when the test point is located outside the SR region.

\section{NUMERICAL RESULTS}

In this section, we present several numerical results obtained by applying the present F-IE formulation. First, the numerical solution is validated by comparison with the exact solution for a perfectly conducting half-plane. Next, numerical results are compared with those obtained from the A-IE method [8] and from a generalized PO method [5].

In order to validate the F-IE method and to provide an estimate of the required size of the SR, the example of a line source close to a perfectly conducting half-plane is considered first. Fig. 6 illustrates the total electric field radiated in the far zone by a line source located in $(x, y)=(L, 0)$ normalized to the radiation pattern of the same line source in free space. The scan angle $\phi$ is that depicted in Fig. 1. The result calculated by the exact solution (dots) is compared with those obtained for a dielectric constant equal to unity with two different numbers of pulse functions. With an SR region of $0.5 \lambda_{0}$ and using ten pulse functions per wavelength (dashed line), the result is quite accurate up to $315^{\circ}$. Increasing the SR to $2.0 \lambda_{0}$ and using 20 expansion functions per wavelength (solid line) allows the F-IE curve 


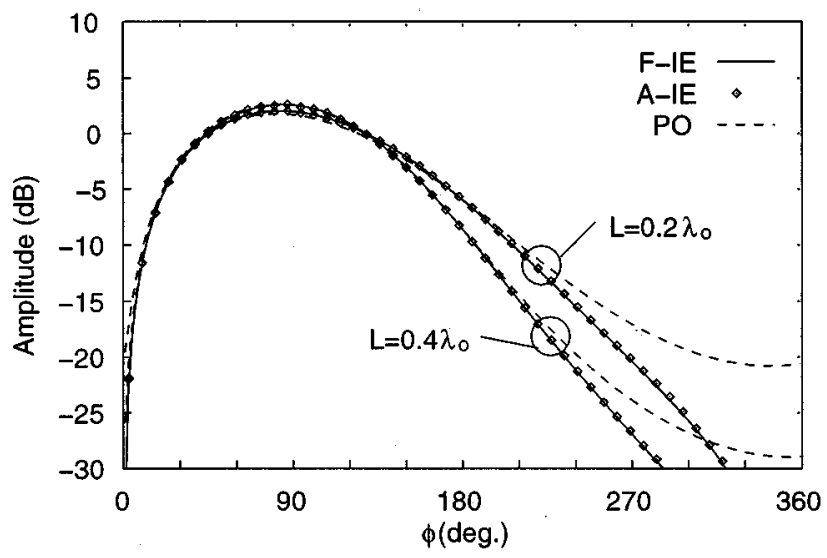

Fig. 7. $L=0.2 \lambda_{0}$ and $0.4 \lambda_{0}, h=0.1 \lambda_{0}, \epsilon_{r}=2.2$.

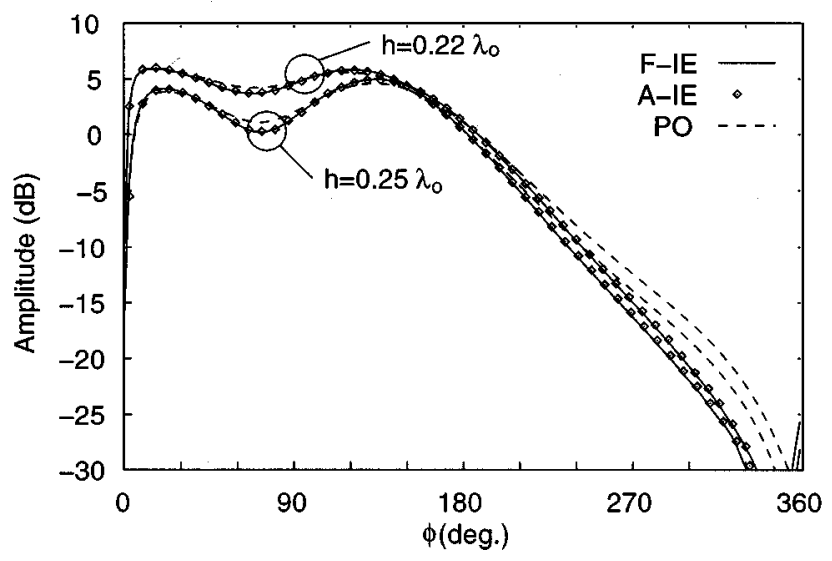

Fig. 8. $L=0.5 \lambda_{0}, h=0.22 \lambda_{0}$ and $0.25 \lambda_{0}, \epsilon_{r}=2.2$.

to superimpose with the exact solution. This choice is used for all the presented results herein after.

Fig. 7 shows the total normalized electric field radiated by a line source on a truncated grounded dielectric slab with $\epsilon_{r}=2.2$ and $h=0.1 \lambda_{0}$. In this case, no guided waves are supported by the slab. Calculations have been made for two different positions of the source ( $L=0.2 \lambda_{0}$ and $\left.L=0.4 \lambda_{0}\right)$. Very good agreement is observed between the F-IE (solid line) and the A-IE (dots), as expected. The PO result (dashed line) compares very well with the exact curves, except for observation in the deep shadow region, where a vanishing field is expected. This is a consequence of the fact that the PO formulation does not account for the proper boundary condition on the bottom face of the ground plane.

The dominant SW mode is propagating when $h>$ $\left(\lambda_{0} / 4\right) / \sqrt{\epsilon_{r}-1}$, and Fig. 8 shows two calculations for $h$ just below $\left(h=0.22 \lambda_{0}\right)$ and above $\left(h=0.25 \lambda_{0}\right)$ the critical thickness associated to cutoff $\left(h_{c}=0.228 \lambda_{0}\right)$. Again, the solutions relevant to the two IE techniques exactly overlap except very close to the bottom face of the ground plane $\left(357^{\circ}\right)$, where a small discrepancy can be observed. Note that this only occurs at very low field levels and is due to the fact that the representation (17) is no longer valid close to the cutoff of an $\mathrm{SW}$, as pointed out in Section IV. To overcome this problem, a proper Fresnel transition function must be incorporated in

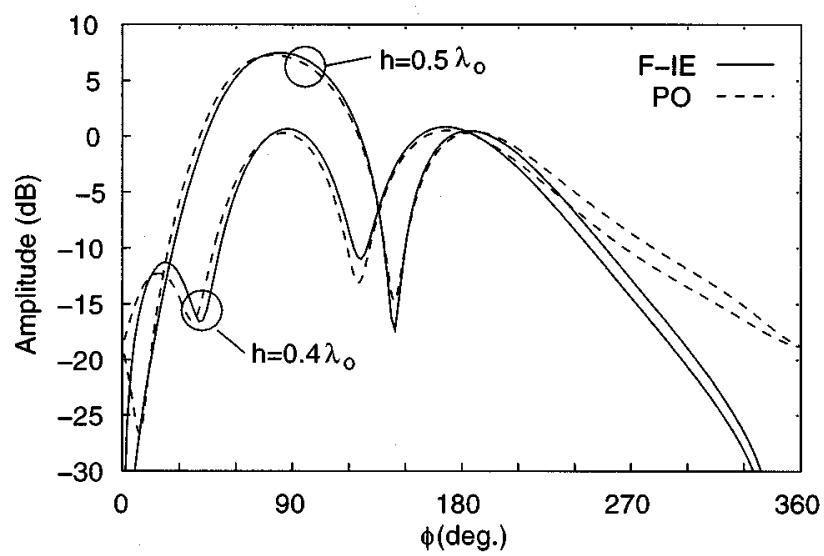

Fig. 9. $L=0.5 \lambda_{0}, h=0.4 \lambda_{0}$ and $0.5 \lambda_{0}, \epsilon_{r}=2.2$.

the entire domain basis functions, or a larger SR can be used. Note that the A-IE is not affected by this problem, since the entire domain basis functions on the aperture do not contain SW contributions, the latter being exponentially attenuated there. In Fig. 9, calculations are reported for slab thicknesses of $0.4 \lambda_{0}$ and $0.5 \lambda_{0}$. As the thickness increases, the dominant surface wave is more strongly excited. This results in a gradual increasing of the relative amplitude of the par-axial lobe, i.e., at $\phi=180^{\circ}$, caused by surface wave diffraction. Also, in these cases the PO is satisfactory up to $\phi=270^{\circ}$.

The most important feature of the F-IE procedure consists of the fact that the number of unknowns is independent from the source position. This allows for treating array problems without further numerical efforts. Indeed, the PO current on D [see Fig. 5(a)] from each line source is obtained from the current of a single line source except for a linear change of variable. Fig. 10 shows the radiation pattern of an array of ten line sources with a source spacing of $0.2 \lambda_{0}$ and a distance $L=0.5 \lambda_{0}$ from the first source to the truncation. Results have been obtained for two different slab thicknesses $\left(h=0.2 \lambda_{0}\right.$ : no SW; $h=0.3 \lambda_{0}$ : one $\mathrm{SW}$ ). A satisfactory agreement with the PO solution is found, especially when SWs are not excited.

\section{CONCLUSION}

A fringe integral equation solving procedure has been presented for describing the scattering by a semi-infinite grounded dielectric slab illuminated by an arbitrary incident $\mathrm{TM}_{z}$ polarized field. The formulation has been obtained by subtracting from the surface/surface integral equations pertinent to the semi-infinite slab, an auxiliary set of equations associated to the canonical problem of an infinite slab illuminated by the same incident field. The application of this process leads us to define expansion functions of the unknown fringe currents that are properly shaped to reproduce the physical behavior of the waves diffracted at the truncation. As a consequence, a MoM solution is presented with pulse basis functions localized on a finite region near the edge and semi-infinite domain basis functions far from it. It is found that by solving only for unknowns physically associated to the fringe field-i.e., localized in the vicinity of the truncation - the necessary number of unknowns is rather small and independent of the slab size. Also, this 


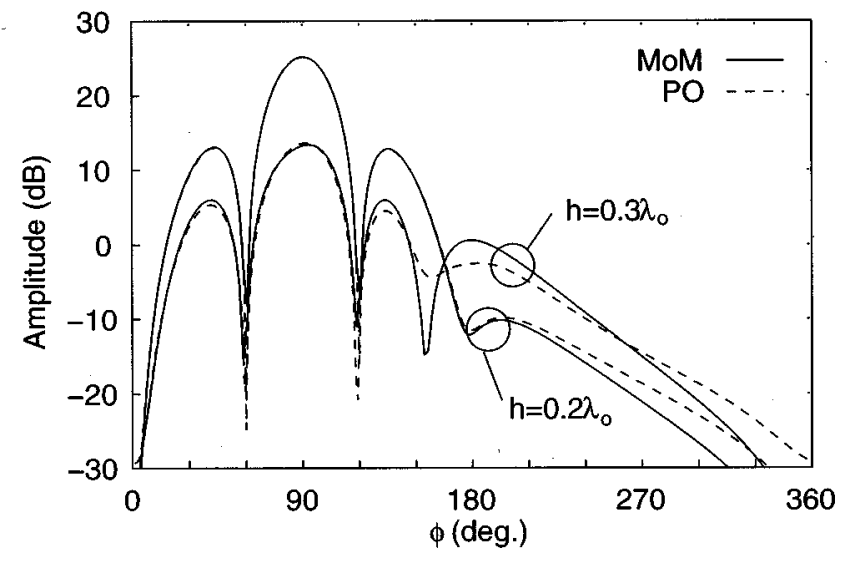

Fig. 10. Array of ten line sources with spacing $0.2 \lambda_{0}, L=0.5 \lambda_{0}, h=0.2 \lambda_{0}$ and $0.3 \lambda_{0}, \epsilon_{r}=2.2$.

method may be easily extended to a truncated slab excited by a $\mathrm{TE}_{z}$ polarized field.

The application of this formulation to the problem of an electric line source placed at the air-dielectric interface has been presented. Numerical results have been obtained and compared with those from the A-IE method and from a generalized PO method. Calculations presented demonstrate that the F-IE method is significantly faster than the A-IE method, with a comparable degree of accuracy.

The present solution may be used as a reference to validate diffraction coefficients, which are developed through approximations (i.e., PO, generalized impedance boundary conditions, etc.). Also, it can be used for problems involving large, but finite, grounded slabs by superimposing the effects of the truncations independently calculated through semi-infinite local structures.

\section{APPENDIX \\ INTEGRAL OPERATORS}

The integral operators defined in Section II are explicitly given here. We let $\boldsymbol{\rho}$ denote the point of observation and $\boldsymbol{\rho}^{\prime}$ the source point. The explicit expression of the free space integral operator for $\boldsymbol{\rho} \in S$ is

$$
\begin{aligned}
\mathbf{E}_{0}[\mathbf{J}, \mathbf{M}] & \\
= & \frac{j}{4} f_{S} \nabla \times\left(H_{0}^{(2)}\left(k_{0}\left|\boldsymbol{\rho}-\boldsymbol{\rho}^{\prime}\right|\right) \mathbf{M}\left(\boldsymbol{\rho}^{\prime}\right)\right) d \boldsymbol{\rho}^{\prime}+\frac{\hat{n}}{2} \times \mathbf{M}(\boldsymbol{\rho}) \\
& -\frac{k_{0} \zeta_{0}}{4} \int_{S} \mathbf{J}\left(\boldsymbol{\rho}^{\prime}\right) H_{0}^{(2)}\left(k_{0}\left|\boldsymbol{\rho}-\boldsymbol{\rho}^{\prime}\right|\right) d \boldsymbol{\rho}^{\prime}
\end{aligned}
$$

where

$$
\begin{array}{ll}
\hat{n} & \text { external normal; } \\
H_{0}^{(2)} & \text { zeroth-order Hankel function of the second } \\
& \text { kind; }
\end{array}
$$

$k_{0}$ and $\zeta_{0} \quad$ wavenumber and the intrinsic impedance of the free space, respectively.
The bar on the integral sign is used to denote a principal value integral, which justifies extraction of one-half of the tangential electric field in (26). The integral operator defined in the interior problem for $\boldsymbol{\rho} \in S$ is

$\mathbf{E}_{1}^{*}[\mathbf{J}, \mathbf{M}]$

$$
\begin{aligned}
= & \frac{j}{4} f_{S+S^{*}} \nabla \times\left(H_{0}^{(2)}\left(k_{1}\left|\boldsymbol{\rho}-\boldsymbol{\rho}^{\prime}\right|\right) \mathbf{M}\left(\boldsymbol{\rho}^{\prime}\right)\right) d \boldsymbol{\rho}^{\prime}-\frac{\hat{n}}{2} \times \mathbf{M}(\boldsymbol{\rho}) \\
& -\frac{k_{1} \zeta_{1}}{4} \int_{S+S^{*}} \mathbf{J}\left(\boldsymbol{\rho}^{\prime}\right) H_{0}^{(2)}\left(k_{1}\left|\boldsymbol{\rho}-\boldsymbol{\rho}^{\prime}\right|\right) d \boldsymbol{\rho}^{\prime}
\end{aligned}
$$

where $k_{1}$ and $\zeta_{1}$ are the wavenumber and the intrinsic impedance of the dielectric, respectively. $S^{*}$ denote the image surface to $S$, and when integrating on this surface, the appropriate sign of the image currents must be chosen according to the image principle.

The Hankel function of zeroth kind contains a logarithmic singularity for $\rho=\boldsymbol{\rho}^{\prime}$, which must be extracted to ensure numerical stability.

\section{REFERENCES}

[1] S. A. Bokhari, J. R. Mosig, and F. E. Gardiol, "Radiation pattern computation of microstrip antennas on finite size ground plane," in Proc. Inst. Elect. Eng. H, vol. 139, 1992, pp. 278-286.

[2] L. Borselli and S. Maci, "Asymptotic, closed-form expressions for the field radiated by patch on a finite substrate," J. Electromagn. Waves Applicat., vol. 11, pp. 689-711, June 1997.

[3] O. M. Bucci and G. Franceschetti, "Electromagnetic scattering by a halfplane with two face impedances," Radio Sci., vol. 11, pp. 49-59, Jan. 1976.

[4] H. C. Ly, G. Rojas, and P. H. Pathak, "Em plane wave diffraction by a planar junction of two thin material half-plane-Oblique incidence," IEEE Trans. Antennas Propagat., vol. 41, pp. 429-441, Apr. 1993.

[5] S. Maci, L. Borselli, and L. Rossi, "Diffraction at the edge of a truncated grounded dielectric slab," IEEE Trans. Antennas Propagat., vol. 44, pp. 863-873, June 1996.

[6] L. S. Andersen, O. Breinbjerg, and J. T. Moore, "The standard impedance boundary condition model for coated conductors with edges: A numerical investigation of the accuracy for transverse magnetic polarization," J. Electromagn. Waves Applicat., vol. 12, pp. 415-446, May 1998.

[7] L. W. Pearson and R. A. Whitaker, "A transverse aperture-integral equation solution for edge diffraction by multiple layers of homogeneous material," Radio Sci., vol. 26, no. 1, pp. 169-174, Jan.-Feb. 1991.

[8] S. Maci, L. Borselli, and A. Cucurachi, "Diffraction from a truncated grounded dielectric slab: A comparative full wave/physical optics analysis," IEEE Trans. Antennas Propagat., vol. 48, pp. 48-57, Jan. 2000.

[9] V. Volski and G. Vandenbosch, "Radiation patterns of sources placed near the truncation of a semi-infinite dielectric structure: The demonstration case of a magnetic line current," IEEE Trans. Antennas Propagat., vol. 48, pp. 240-245, Feb. 2000.

[10] J. R. Mosig and R. C. Hall, "Numerical analysis of microstrip patch antennas," in Handbook of Microstrip Antennas, J. R. James and P. S. Hall, Eds. Stevenage, U.K.: Peregrinus, 1998, ch. 8.

[11] P. A. Ufimtsev, "Elementary edge waves and the physical theory of diffraction," Electromagnetics, vol. 11, no. 2, pp. 125-159, Apr.-June 1991.

[12] B. L. Van der Waerden, "On the method of saddle points," Appl. Sci. Res., vol. B2, pp. 33-45, Jan. 1951.

[13] J. R. Mosig and F. E. Gardiol, "Analytical and numerical techniques in the green's function treatment of microstrip antennas and scatterers," Proc. Inst. Elect. Eng., vol. 130, no. 2, pp. 175-182, Mar. 1983. 


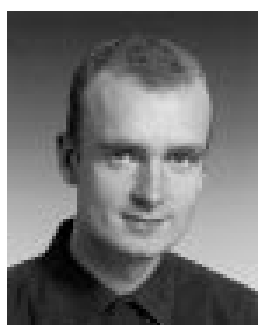

Erik Jørgensen (S'99) was born in Roskilde, Denmark, in 1974. He received the M.S. degree in electrical engineering from the Technical University of Denmark, Lyngby, in 2000, where he is currently pursuing the Ph.D. degree in the area of integral equations.

In fall 1998, he spent five months at the Department of Information Engineering, University of Siena, Italy. His research interests include high-frequency techniques and all areas of computational electromagnetics.

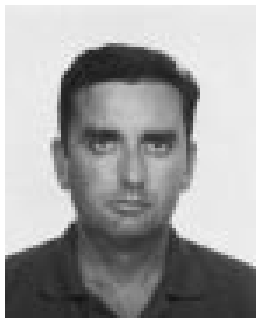

Stefano Maci (M'92-SM'99) was born in Rome, Italy, in 1961. He received the Doctor degree in electronic engineering from the University of Florence, Italy, in 1987.

In 1990, he joined the Department of Electronic Engineering, University of Florence, Italy, as an Assistant Professor. In 1998, he became an Associate Professor of electromagnetism at the University of Siena, Italy. In 1997, he was an invited Professor at the Technical University of Denmark, Copenhagen. Since 1996, he has been involved in projects of the European Space Agency regarding the electromagnetic modeling of antennas. $\mathrm{He}$ is an Associate Editor of the TRANSACTIONS ON ElECTROMAGNETIC COMPATABILITY. His research interests are focused on electromagnetic theory, mainly concerning high-frequency methods for electromagnetic scattering and diffraction. He also developed research activity on microwave antennas, particularly focused on the analysis, synthesis, and design of patch antennas.

Dr. Maci received the national Young Scientists "Francini" award for his Laurea thesis in 1988 and the "Barzilai" prize for the best paper at the national Italian congress of electromagnetism (XI RiNEm) in 1996.

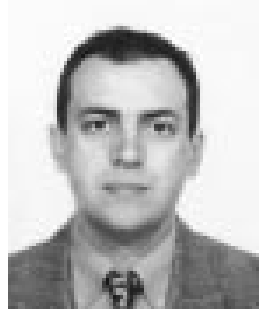

Alberto Toccafondi (S'93-M'94) was born in Prato, Italy, in 1960. He received the Doctor degree (cum laude) in electronic engineering and the Ph.D. degree in electromagnetism from the University of Florence, Italy, in 1989 and 1994, respectively.

Since 1995, he has been an Assistant Professor in the Information Engineering Department, University of Siena, Italy. His research interests are concerned with reflector and lens antennas and with the analytic and numerical techniques for the electromagnetic radiation and scattering. 